\section{Denis McQuail}

\section{Journalism and Society}

London: Sage 2013. - 244 S.

ISBN 978-1-4462-6679-3

Wenn kritische Denker wie Jo Bardoel, Clifford Christians, Stephen Coleman, Mark Deuze, Christina Holtz-Bacha, Gianpietro Mazzoleni und Kaarle Nordenstreng übereinstimmend ein Buch loben, muss es gut sein - auch wenn die Lobpreisungen der Kollegen lediglich im Klappentext des besagten Werkes auftauchen. Denis McQuails Journalism and Society sei für Studierende der Kommunikationswissenschaft und Journalistik ein „must-read“ (Holtz-Bacha). Der "Meister der Synthese“ (Bardoel) präsentiere eine Sozialtheorie des Journalismus, die über den typischen angloamerikanischen Fokus hinausgehe - „a truly global scope" (Bardoel). Der Band zeige nicht nur, „was Journalisten tun und was sie tun sollten" (Coleman), sondern liefere zudem die „theoretische Basis eines neuen soziologischen Paradigmas öffentlicher Kommunikation in einer stark veränderten Medien- und Informationsumwelt" (Mazzoleni). Und, nicht zuletzt, sei das Werk sehr gut didaktisch aufbereitet ein „beispielhaftes Lehrbuch“ (Nordenstreng).

Nach der Lektüre der 220 Textseiten teile ich den Enthusiasmus der Kollegen - mit Abstrichen. Tatsächlich hat McQuail Journalism and Society inhaltlich, formal und stilistisch als Lehrbuch angelegt. Er versteht es als ,eine Einführung in die Sozialtheorie des Journalismus" (Vorwort), vor allem in die "Theorie der Beziehung von Journalismus und Gesellschaft" (1). Unter verschiedenen Theorietypen (Sozialtheorien, Wirtschaftstheorien, politische Theorien usw.) sei die Sozialtheorie („social theory") für das Verständnis der öffentlichen Aufgabe des Journalismus am relevantesten. Sozialtheorie sei eine "Mischung aus Deskription und normativer Präskription" (9-10) mit drei Hauptvarianten: Marktliberalismus, Professionalismus und Demokratietheorien (51-52).

Den Terminus „Sozialtheorie“ verwendet McQuail insofern eher diffus als Sammelbegriff für jene traditionellen Ansätze, die in der zweiten Hälfte des 20. Jahrhunderts den intellektuell vergleichsweise bescheidenen anglo-amerikanischen Diskurs über die Beziehungen von Journalismus und Gesellschaft geprägt haben. Weitgehend unberücksichtigt bleiben hingegen Überlegungen aus dem Umfeld der Cultural Studies, obgleich diese u. a. von John Hartley, Stuart Allen oder Barbara Zelizer intensiv dis- kutiert wurden und in der internationalen Journalismusforschung wirkmächtig sind. Keine Erwähnung findet auch die seit den 1970er Jahren im deutschen Sprachraum geführte, primär soziologisch inspirierte Debatte über den Journalismus als System, Handlungszusammenhang, soziales Feld oder systembezogene Akteurkonstellation, abgesehen von einem kurzen Verweis auf Luhmanns „Realität der Massenmedien".

Gleichwohl führt McQuail einige basale soziologische Kategorien zur Beschreibung des Journalismus ein, ohne allerdings deren theoretische Wurzeln zu benennen. Um Schlüsselaspekte wie die journalistische Freiheit oder die Verpflichtung des Journalismus gegenüber anderen zu verstehen, sei es beispielsweise sinnvoll, drei Analyseebenen zu unterscheiden: „die Gesellschaft; die Nachrichtenorganisation; und der individuelle Journalist“ (5). Die meisten Fragen der Journalismustheorie, die einen Bezug zur Gesellschaft haben, seien mit Hilfe dieser Differenzierung zu behandeln. Jedoch sei es notwendig, eine weitere Perspektive einzubeziehen - „die der Leser und des Publikums“ (8). Darüber hinaus variiere Journalismus und seine Analyse entlang weiterer Dimensionen: Autorenschaft versus Beschäftigung, Profession versus Handwerk oder Kunst, lokal versus national und kosmopolitisch, Profit versus non-profit, Generalist versus Spezialist, kontradiktorisch und aktiv versus unterstützend oder neutral, Print versus andere Medien, informativ versus unterhaltend (12).

Diese Differenzierungen sowie die von ihm als Sozialtheorie bezeichneten Ansätze zur Beschreibung der Beziehungen von Journalismus und Gesellschaft nutzt McQuail als Ausgangspunkte, um die ihn interessierenden Kernfragen nach der Verantwortung (Kapitel 2), den Prinzipien (Kapitel 3), den Funktionen (Kapitel 5), den Strukturen (Kapitel 6) und der Rechenschaftspflicht des Journalismus (Kapitel 7) zu beantworten. Im vierten Kapitel, das aus meiner Sicht besser an die Einleitung angefügt worden wäre, entwickelt er einen „Rahmen zur Analyse von Journalismus“ (73), der im Kern auf den institutionalisierten und professionellen Journalismus ausgerichtet ist, welcher im 19. Jahrhundert entstand und seine Blüte im 20. Jahrhundert erlebte. Eine Orientierung an den Strukturen und Funktionen des traditionellen Journalismus sei aber weiterhin sinnvoll, weil neue Entwicklungen vergleichend besser zu identifizieren seien.

Konsequenterweise diskutiert McQuail eine Vielzahl aktueller Herausforderungen, welche die Beziehungen von Journalismus und Gesell- 
schaft beeinflussen und gegebenenfalls verändern. Die Benennung, Vereinfachung und gelegentliche Zuspitzung journalismusbezogener Problemfelder stärkt den Lehrbuchcharakter des Bandes. McQuail entwickelt dabei keine simplifizierenden Synthesen, sondern stellt oftmals unterschiedliche oder gar konträre Überlegungen gegenüber - etwa marktliberale und demokratietheoretische Ansätze. Im achten Kapitel problematisiert er ausführlicher die Konsequenzen veränderter Medientechnologien für Journalismus, Medieninstitutionen und deren Beziehungen zur Gesellschaft. Ein „neues soziologisches Paradigma öffentlicher Kommunikation in einer stark veränderten Medienund Informationsumwelt" (Mazzoleni) konnte ich dabei zwar nicht ausmachen - für ein Einführungsseminar zum Thema „Journalismus und Gesellschaft" bietet jedoch auch dieser Abschnitt hinlänglich Stoff.

Insgesamt liefert McQuail mit Journalism and Society eine Bestandsaufnahme der Beziehungen von Journalismus und Gesellschaft, welche die komplexen Zusammenhänge vereinfacht, ohne sie zu trivialisieren. Normative Aspekte thematisiert er dabei besonders ausführlich. Wie bei seinem erfolgreichen Klassiker Mass Communication Theory konzentriert sich McQuail auf Kernthemen, insbesondere auf die Leistungen, Funktionen und Verpflichtungen des Journalismus westlicher Demokratien gegenüber der Gesellschaft, differenziert soweit nötig, verzichtet auf kleinteilige Verweise und gibt kapitelweise Empfehlungen für die vertiefende Lektüre. Anders als Kollege Bardoel kann ich dem Band zwar keine "wirklich globale“ Orientierung bescheinigen (Probleme wie u. a. Korruption, politische Morde oder elitäre Eigentumsverhältnisse werden nicht behandelt). Unterm Strich eignet sich Journalism and Society jedoch sehr gut als Lehrbuch für BachelorStudierende der Kommunikationswissenschaft und Journalistik.

Martin Löffelholz

\section{Wiebke Möhring / Daniela Schlütz (Hrsg.) \\ Handbuch standardisierte \\ Erhebungsverfahren in der \\ Kommunikationswissenschaft \\ Wiesbaden: Springer VS, 2013 - 389 S.}

ISBN 978-3-531-18775-4

Wiebke Möhring und Daniela Schlütz haben das Handbuch mit dem Ziel herausgegeben, „die Möglichkeiten und Varianten quantitativempirischer Forschung umfassend und syste- matisch darzustellen und dabei die wichtigsten Methodenentwicklungen in der Kommunikationswissenschaft aufzuzeigen" (S. 9). Ein weiteres Handbuch, das nicht-standardisierte, qualitative Erhebungsverfahren der Kommunikationswissenschaft vorstellt, sei in Planung. Das Buch richte sich, so die Herausgeberinnen, an Kolleginnen und Kollegen, die sich einen Überblick über standardisierte Erhebungsverfahren verschaffen oder einzelne Verfahren kennenlernen wollen.

Die Herausgeberinnen haben sich entschieden, dem Handbuch eine Einleitung voranzustellen, in der zunächst ethische Fragen empirischer Forschung behandelt werden. Das erste Kapitel ist den methodologischen Grundlagen gewidmet. Rinaldo Kühne erläutert die wesentlichen Teilschritte des standardisierten Forschungsprozesses, des Messens (inklusive der klassischen Testtheorie), der Skalierung und weist im Ausblick auf die Bedeutung latenter Variablenmodelle hin. Tilo Hartmann und Leonard Reinecke beschreiben in ihrem Teilkapitel die typischen Schritte der Konstruktion einer Skala. Olaf Jandura und Melanie Leidecker geben einen Überblick über Verfahren der Stichprobenbildung. Armin Scholl umreißt in seinem Beitrag das Problem der Reaktivität, seiner methodischen Konsequenzen und diskutiert den forschungspraktischen Umgang dieser Problematik.

Das zweite Kapitel umfasst Beiträge zu verschiedenen Forschungsdesigns. Helmut Scherer und Teresa K. Naab arbeiten die Unterschiede zwischen Trend- und Paneldesign heraus und gehen mit Blick auf das Paneldesign ausführlich auf die Spezifika der Tagebuchmethode ein. Christoph Klimmt und René Weber stellen die verschiedenen Varianten des Experiments vor und beschreiben die speziellen methodischen Herausforderungen der experimentellen Medienforschung. Birgit Stark und Melanie Magin stellen die Grundprinzipien komparativer Forschung vor. Anja Kalch und Helena Bilandzic skizzieren die Möglichkeiten und Grenzen von Mehrmethodendesigns und gehen beispielhaft auf die verschiedenen im Fach eingesetzten Methodenkombinationen und die empirischen Erträge dieser Studien ein.

Die folgenden Kapitel, in denen es um die Methoden der Befragung, Inhaltsanalyse und Beobachtung geht, beginnen jeweils mit einem Grundlagenbeitrag. Das dritte Kapitel leiten die Herausgeberinnen selbst ein: Sie stellen die Grundprinzipien einer standardisierten Befragung vor, grenzen unterschiedliche Modi der Erhebung voneinander $\mathrm{ab}$ und gehen auf handwerkliche Aspekte der Fragebogenkonstrukti- 